\title{
An Inductive Algorithm to Construct Finite Lattices
}

\author{
By Shoji Kyuno
}

\begin{abstract}
G. Birkhoff [1] proposed the following problem: Enumerate all finite lattices which are uniquely determined (up to isomorphism) by their diagram, considered as a graph.

It is not known how many lattices of order $n$ exist, except when the value of $n$ is quite small. The aim of this note is to give an algorithm to construct inductively all finite lattices of order $n$. Using this algorithm, we have found that there exist 222 lattices for $n=8$ and 1078 lattices for $n=9$. All lattices of order $n \leqslant 8$ are shown at the end of this note.
\end{abstract}

1. A Graphical Representation. Since a lattice is an ordered set, it can be represented by a Hasse diagram. To represent a lattice here, we transform slightly its usual Hasse diagram, writing one of the longest chains straight up, and we "locate all elements upward closely." To "locate all elements upward closely" means that for any element $a$, except the maximal element, there exists at least one element which dominates $a$ and is one level above $a$, where a level means the set of all points having equal $Y$-coordinates. Such a diagram is briefly called a "diagram closed upward."

Example. A lattice having the chains $f<e<d<c<b<a, f<h<d$, $f<h<$ $g<b$, and $e<i<a$, is represented in the following diagram closed upward:

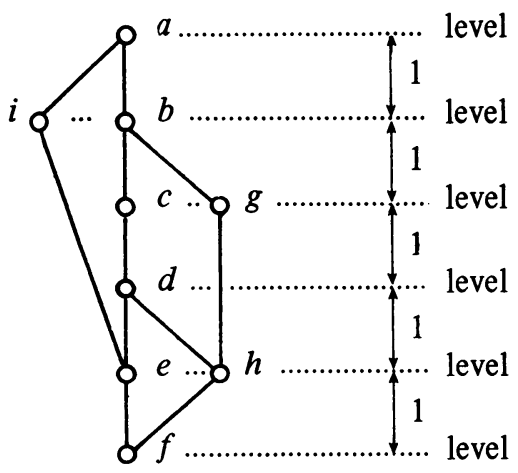

Figure 1

In Figure $1, a$ is the maximal element. For any element $x$ except $a$ we can find an element which dominates $x$ with one level-difference.

The following theorem assures the possibility of representing a lattice by such a diagram closed upward.

THEOREM 1. A lattice $L$ can be represented by a diagram closed upward.

Received March 2, 1977.

AMS (MOS) subject classifications (1970). Primary 06A20, 05C30, $02 \mathrm{E} 10$. 
Proof. First make a Hasse diagram of a given lattice $L$. Let $E(L)$ be the element set of $L$. Denote the maximal element by $m$. Let the elements dominated by $m$ be $a_{1}, a_{2}, \ldots, a_{s}$. Let the elements dominated by $a_{i}(i=1,2, \ldots, s)$ be $b_{i j(i)}(i=1$, $\left.2, \ldots, s ; j(i)=1,2, \ldots, t_{i}\right)$.

We now transform the Hasse diagram into a diagram closed upward. Locate $a_{1}$, $a_{2}, \ldots, a_{s}$ at one level downward from $m$ keeping invariant all connection relations. For an element $b_{i j(i)}$, if

$$
\left\{x \in E(L) \mid b_{i j(i)} \leq x\right\} \subseteq\left\{m, a_{i} ; i=1,2, \ldots, s\right\},
$$

then we replace $b_{i j(i)}$ by $a_{i j(i)}$; otherwise we leave $b_{i j(i)}$ standing. Do this test for all $b_{i j(i)}$ 's and locate all $a_{i j(i)}$ 's at one level downward from the level of $a_{i}$ 's, keeping invariant all connection relations.

The set of $a_{i j(i)}$ 's is not empty. For if $b_{11}$ is one of the elements left alone, let one chain which is not a subset of $\left\{m, a_{i} ; i=1,2, \ldots, s\right\}$ be the following:

$$
b_{11} \lesseqgtr \cdots \leqq b_{p q} \lesseqgtr a_{r} \lesseqgtr m \text {. }
$$

If there is no chain upward from $b_{p q}$ except $b_{p q} \lessgtr a_{r} \lessgtr m$, then we can replace $b_{p q}$ by $a_{p q}$, and our statement will then be true. Hence, we assume that there exists the following chain upward from $b_{p q}$ :

$$
b_{p q} \nrightarrow \cdots \lesseqgtr b_{u v} \lessgtr a_{w} \lessgtr m .
$$

If there is no chain from $b_{u v}$ except $b_{u v} \lesseqgtr a_{w} \lesseqgtr m$, then our statement will be true. By continuing this argument, we obtain the following chain:

$$
b_{11} \lesseqgtr b_{p q} \lesseqgtr b_{u v} \lesseqgtr \cdots \lesseqgtr a_{m} \lesseqgtr m .
$$

However, the finiteness of $b_{i j(i)}\left(i=1,2, \ldots, s ; j(i)=1,2, \ldots, t_{i}\right)$ shows that the length of the above chain must be finite. Therefore, our statement is true.

Repeat the same procedures for the elements dominated by all $a_{i j(i)}$ 's and so on. By the finiteness of the lattice we get to the minimal element. We draw one of the longest chains straight, and thus obtain the diagram. This completes the proof.

In the following we mean by 'lattice' the diagram closed upward.

2. To Construct the Lattice. Construction of all lattices of given order $n$ will be accomplished by an inductive method. For this we prepare the following theorem:

THEOREM 2. From an n-element lattice we can construct an $(n-1)$ element lattice by removing an element dominated by the maximal element and by rearranging the rest to make a lattice.

Proof. Let $m$ be the maximal element of a given $n$-element lattice. Let $a_{0}$ be an element dominated by $m$. Let $a_{i}(i=1,2, \ldots, s)$ be the elements dominated by $a_{0}$.

For each $i \in\{1,2, \ldots, s\}$, if there exists at least another chain upward from $a_{i}$ except $a_{i} \lesseqgtr a_{0} \leq m$, then take out the line segment connecting $a_{0}$ to $a_{i}$. If there exists no other chain upward except $a_{i} \leq a_{0} \leq m$, then take out the line segment connecting $a_{0}$ to $a_{i}$ and connect $m$ with $a_{i}$ by a new line segment. At last, take out the element $a_{0}$ and the line segment connecting $m$ with $a_{0}$. Then we can obtain a lattice of order $(n-1)$. 
From Theorem 2, we can see how to construct the set of all lattices of order $n$. It will be derived from the set of all lattices of order $(n-1)$ by applying the inverses of all possible procedures to make an $(n-1)$-element lattice from an $n$-element lattice, as stated in the proof of Theorem 2 .

In the following, we show the detail of the procedure to construct the set of all lattices of order $n$.

Algorithm to produce from an $(n-1)$-element lattice $L$ a set of $n$-element lattices:

Let $E(L)$ be the element set of $L$.

Let $m$ be the maximal element of $L$.

Let the elements dominated by $m$ be $a_{1} \cdots a_{k}$.

Let the elements of $E(L)-\left\{m, a_{1}, \ldots, a_{k}\right\}$ be $e_{1} \cdots e_{l}$.

We shall produce a list of $n$-element lattices, $\operatorname{LIST}(n)$.

1. Let $\operatorname{LIST}(n)=\varnothing$.

2. In $L$, add an element $a_{0}$ which dominates $m$. Add this lattice to $\operatorname{LIST}(n)$.

3. If $k>1$ do:

For each $q$-subset $s_{1} \cdots s_{q}$ of $a_{1} \cdots a_{k}$, in turn for $1 \leqslant q<k$, do:

for all $s_{i}$, let $m$ no longer dominate $s_{i}$.

for all $s_{i}$, let $a_{0}$ dominate $s_{i}$.

Let $m$ dominate $a_{0}$.

For each result obtained above, check for all $a_{j}$ of $\left\{a_{1}, \ldots, a_{k}\right\}-\left\{s_{1}, \ldots, s_{q}\right\}$ whether all $a_{0} \wedge a_{j}$ are unique, where $a_{0} \wedge a_{j}$ denotes the g.l.b. of $a_{0}$ and $a_{j}$. Append any result for which all $a_{0} \wedge a_{j}$ are unique to $\operatorname{LIST}(n)$.

4. For each $e_{i}(1 \leqslant i \leqslant l)$ do:

Let $m$ dominate $a_{0}$.

Let $a_{0}$ dominate $e_{i}$.

Append the result to $\operatorname{LIST}(n)$.

5. For each lattice $M$ except the one obtained in 2 in the list $\operatorname{LIST}(n)$ created thus far, do:

Let $a_{0}, x_{1} \cdots x_{m}$ be the elements dominated by $m$ in $M$.

Let $L_{a_{0}}=\left\{a_{i} \in E(M) \mid a_{i} \lesseqgtr a_{0}\right\}$.

Let $P=E(M)-L_{a_{0}}-\left\{m, a_{0}, x_{1}, \ldots, x_{m}\right\}$.

Let $p_{1} \cdots p_{t}$ be the elements of $P$.

For each $p_{i}(1 \leqslant i \leqslant t)$ do:

If $a_{0} \wedge p_{i}$ is not dominated by $a_{0}$, and moreover if for any $a_{j} \in L_{a_{0}}$, $a_{j} \bigvee p_{i}=m$ or $a_{j} \bigvee p_{i}=p_{i}$, then let $a_{0}$ dominate $p_{i}$ and append this lattice to $\operatorname{LIST}(n)$.

6. For each lattice $M$ newly constructed in 5 in the list LIST( $n)$, do: the same procedures stated in 5 .

7. For each lattice constructed in 6 in the list $\operatorname{LIST}(n)$, do: the same procedures stated in 5 .

8. Repeat the same procedures stated as in 7 for each lattice $M$ newly constructed in the previous step until the set $P$ becomes empty. 
3. A Matrix Representation. To find all isomorphic lattices we consider a matrix representation. For a given diagram closed upward, denote the maximal element by 1 and number the levels $1,2,3, \ldots$, from the top to bottom. Denote the elements on the same level by the same numbers and distinguish different elements by the suffixes $a, b, c, \ldots$.

\section{Examples.}

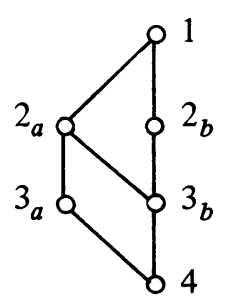

Figure A

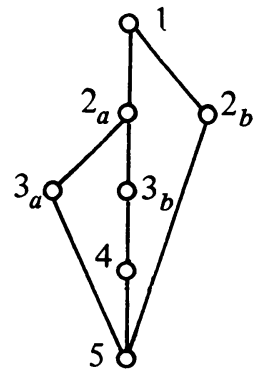

FIGURE B

Make a matrix whose columns represent all chains from the maximal element to the minimal element. Put 0's between two nonadjacent integers to make the lengths of all columns the same.

Examples. Matrices corresponding to Figures A and B.

$$
A=\left(\begin{array}{ccc}
1 & 1 & 1 \\
2 & 2 & 2 \\
a & 2_{b} \\
3 a & 3_{b} & 3_{b} \\
4 & 4 & 4
\end{array}\right), \quad B=\left(\begin{array}{ccc}
1 & 1 & 1 \\
2 & 2 a & 2 b \\
3 & 3_{b} & 0 \\
0 & 4 & 0 \\
5 & 5 & 5
\end{array}\right)
$$

We may define the equality of two such $l \times m$-matrices $P, Q$ as follows:

If some permutation of all columns of $P$ and some replacements of all suffixes on each row of $P$ transform $P$ to $Q$, then we say that $P$ and $Q$ are equal.

By this definition, for a lattice we have a unique matrix representation. Conversely, if a matrix is given, the suffixes of each row determine the elements which should be located on the corresponding level, and each column shows which element should be connected with which element. Thus a matrix determines a unique lattice.

Acknowledgements. The author wishes to thank the referee for his valuable suggestions and in particular for giving a concise description of the algorithm.

\section{LATTICES}

$$
n=1 \quad n=2 \quad n=3
$$
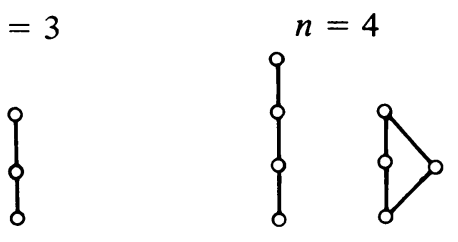
$n=5$
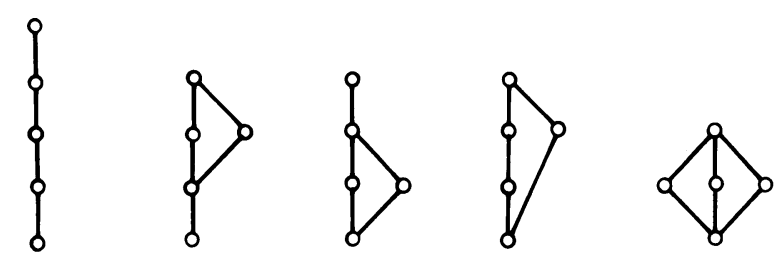

$n=6(15$ pieces $)$
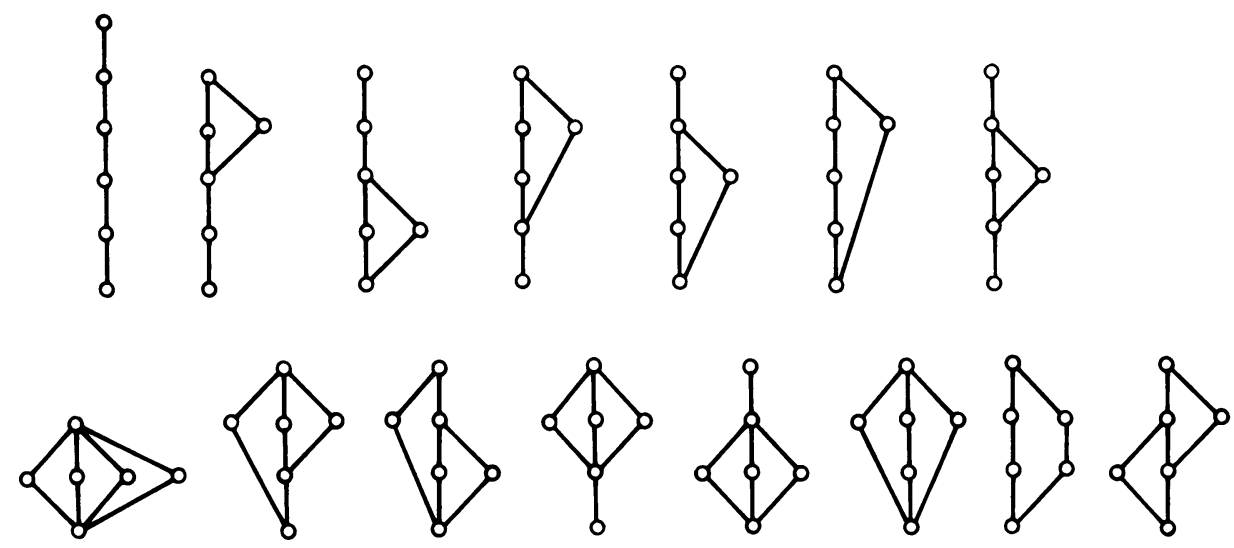

$$
n=7 \text { (53 pieces) }
$$
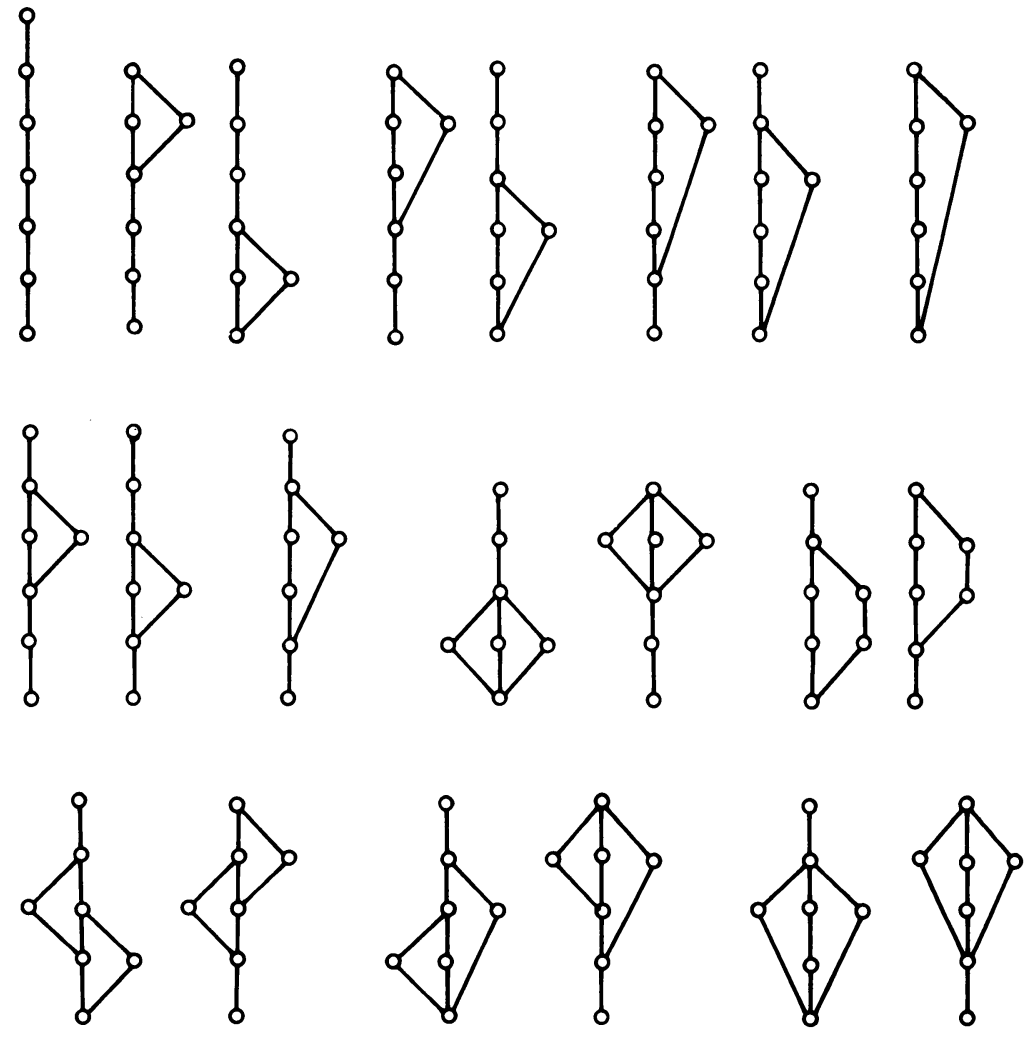


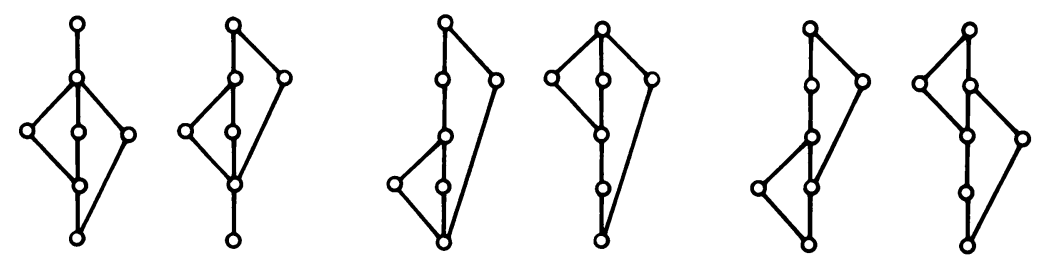

\$

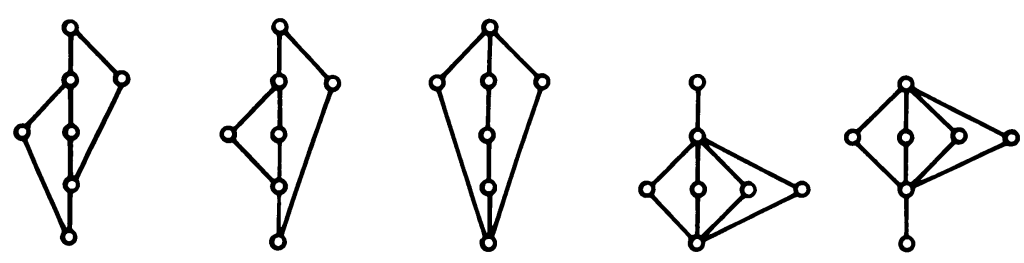

全

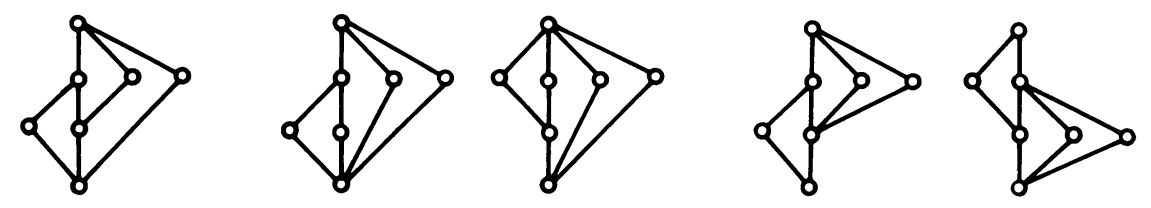

$\$$ 


$$
n=8 \text { (222 pieces) }
$$
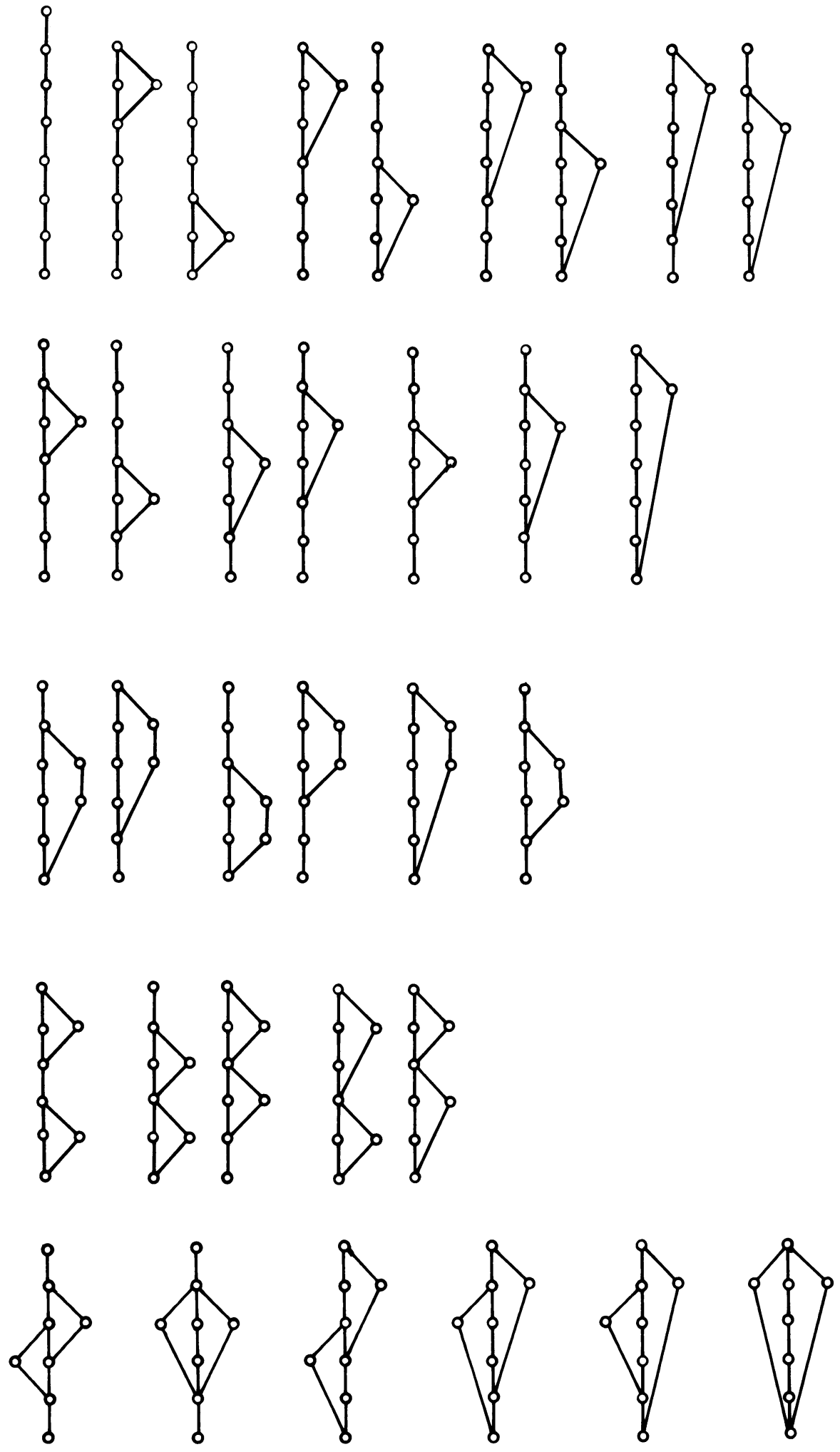

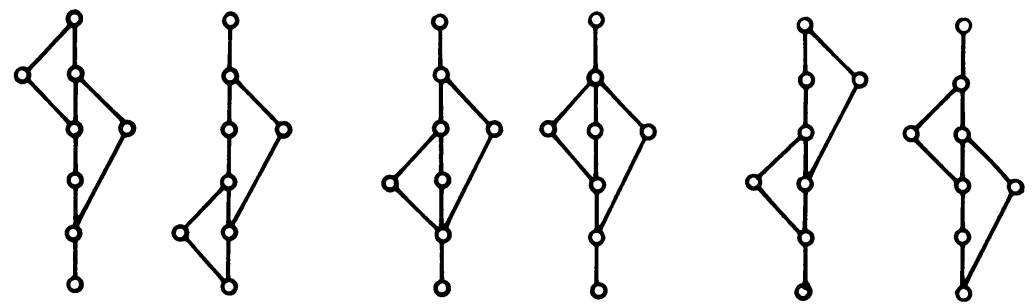

$\$$
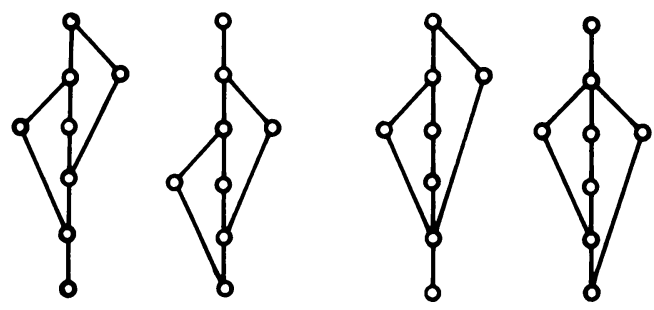

引
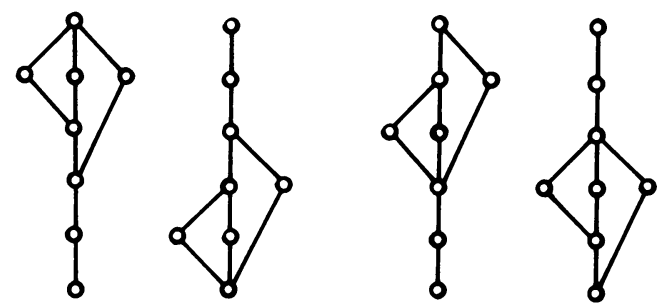

20?
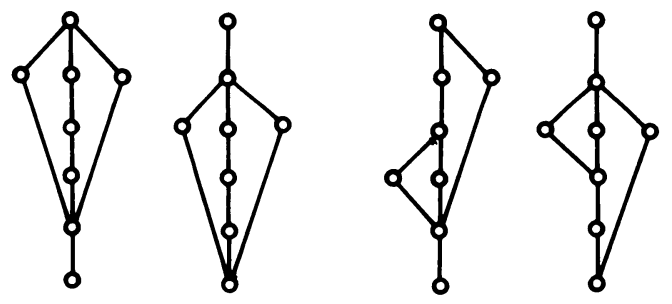

ई
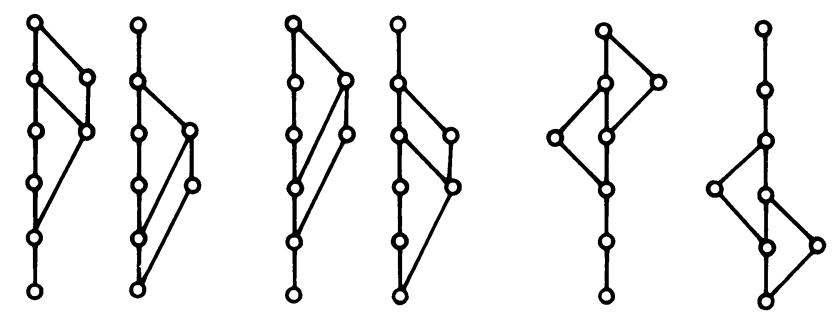

?
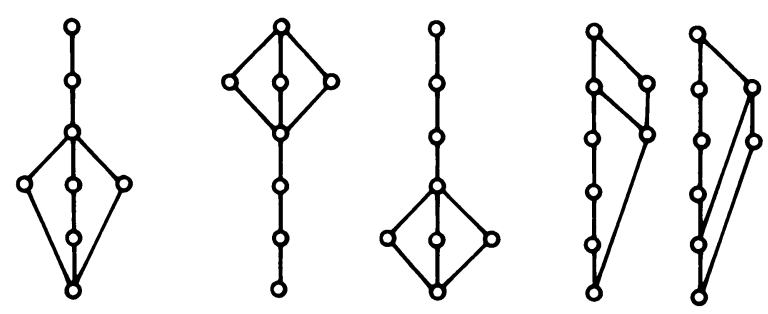

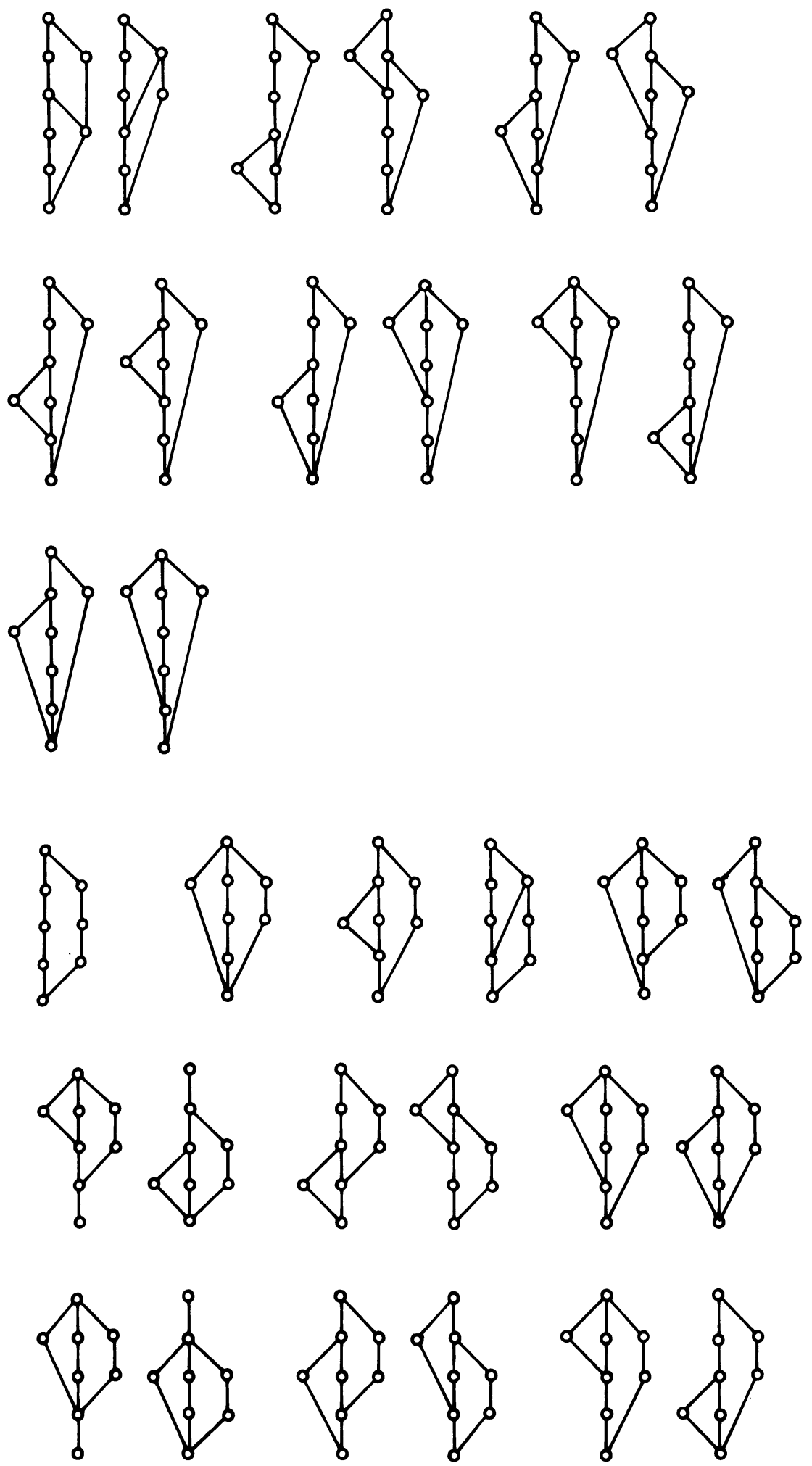
卓

ऐ)

$\$$

$\forall$ \$

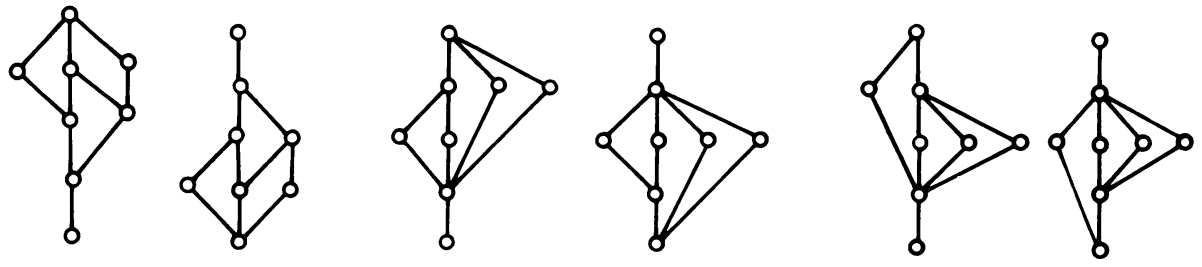

\&?

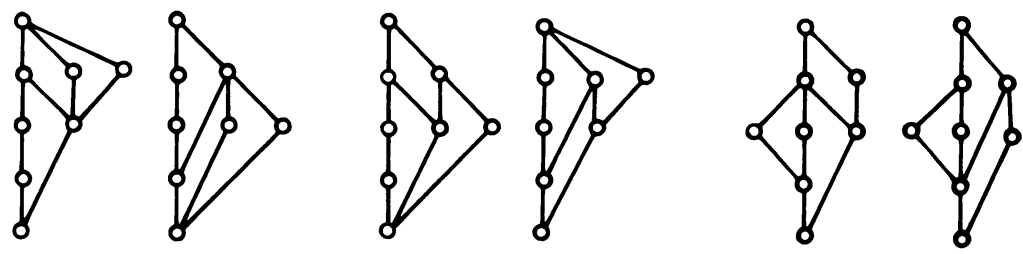


\&

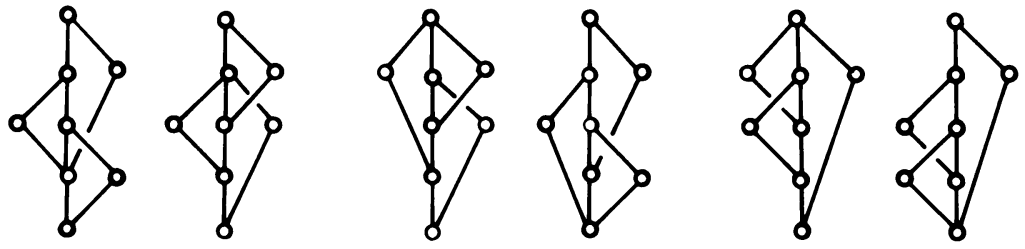

\$ की

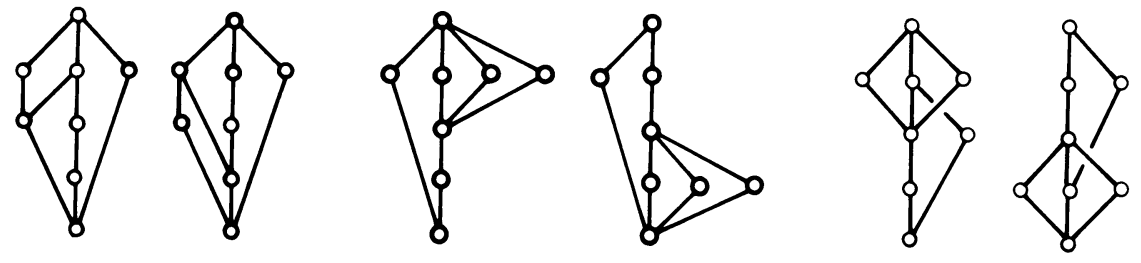

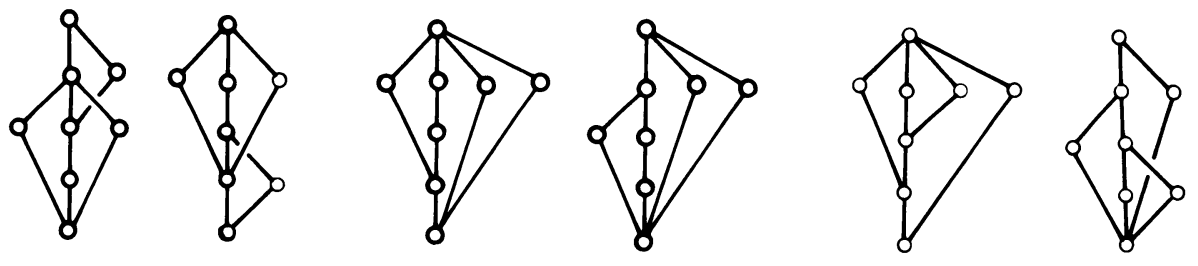
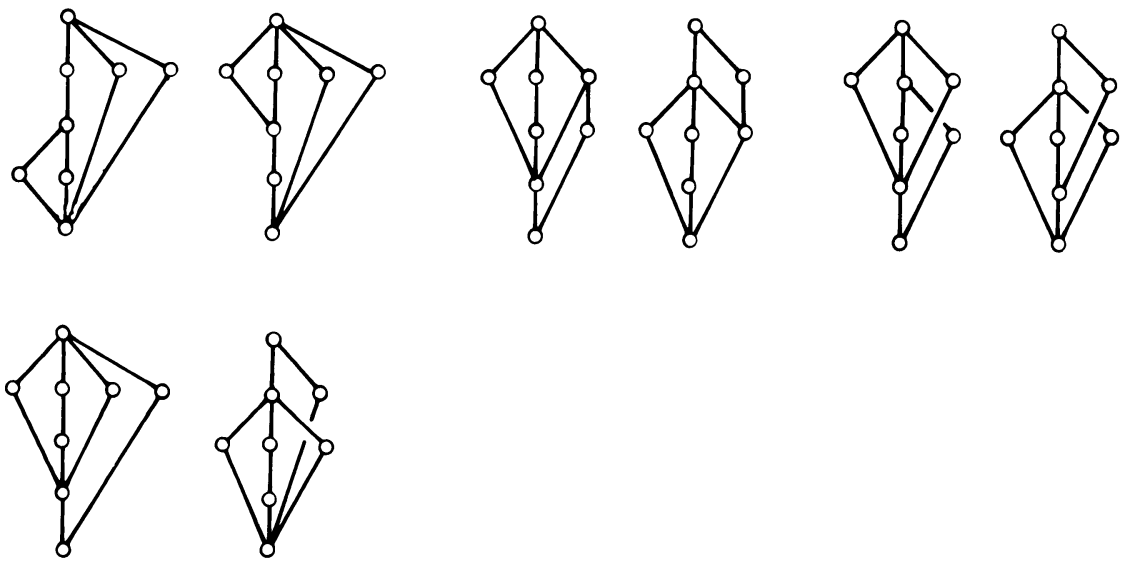


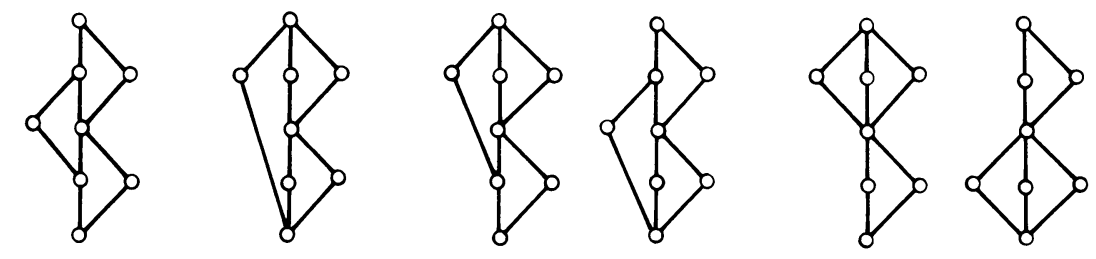
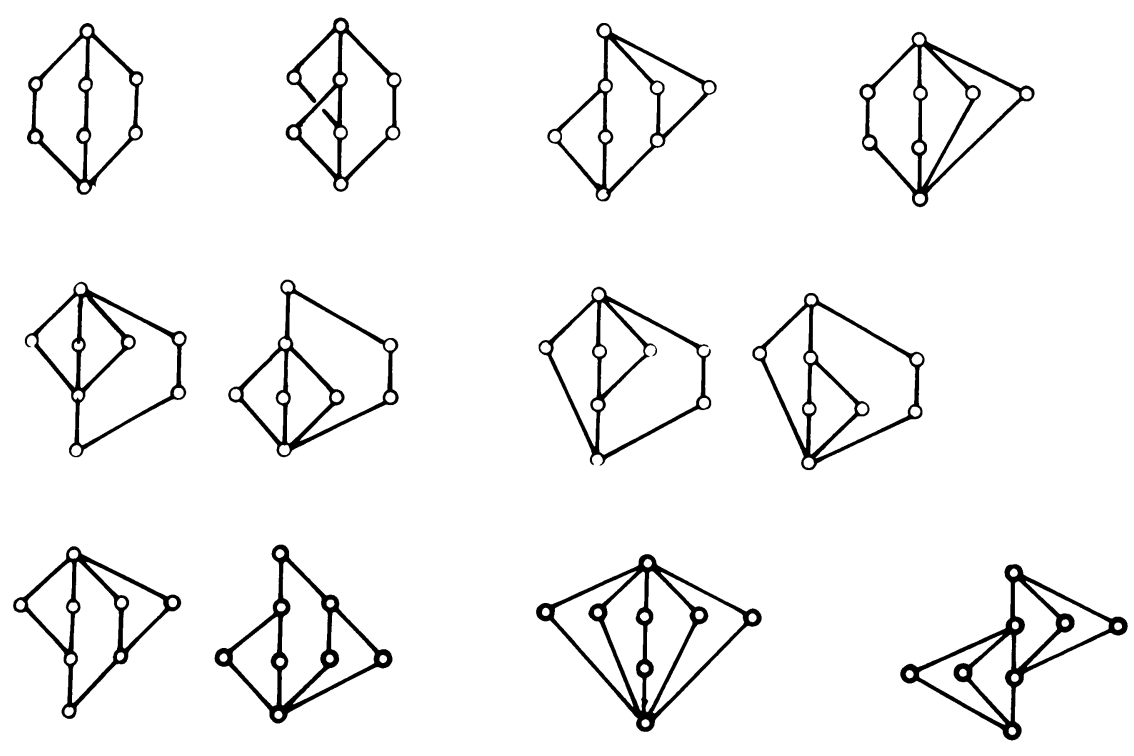

\&
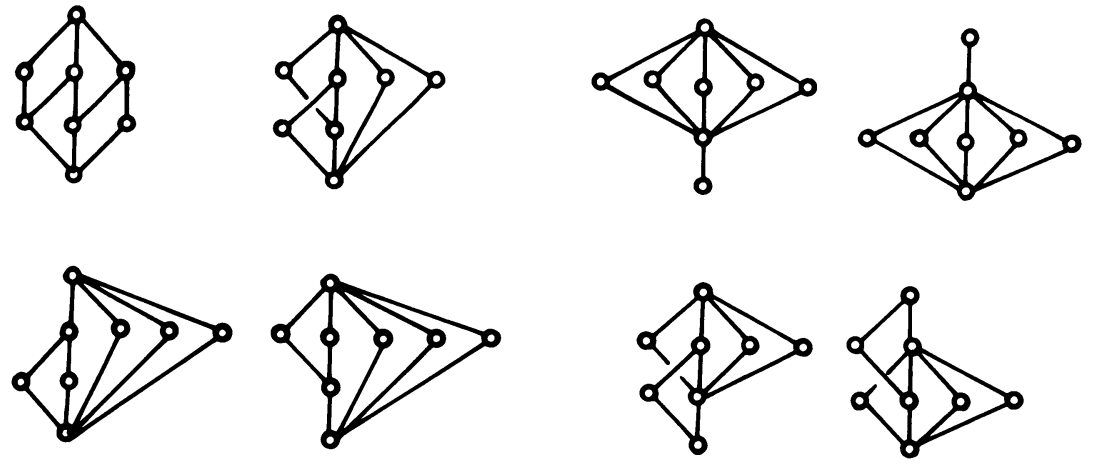

皮

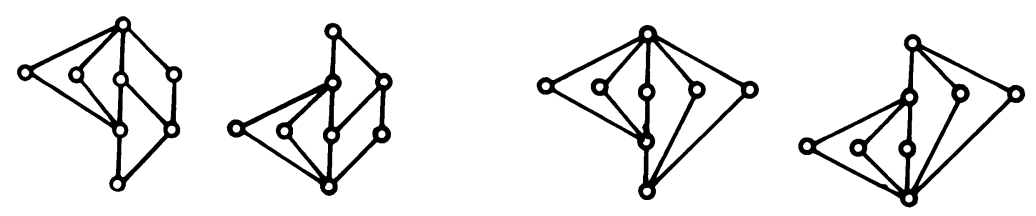



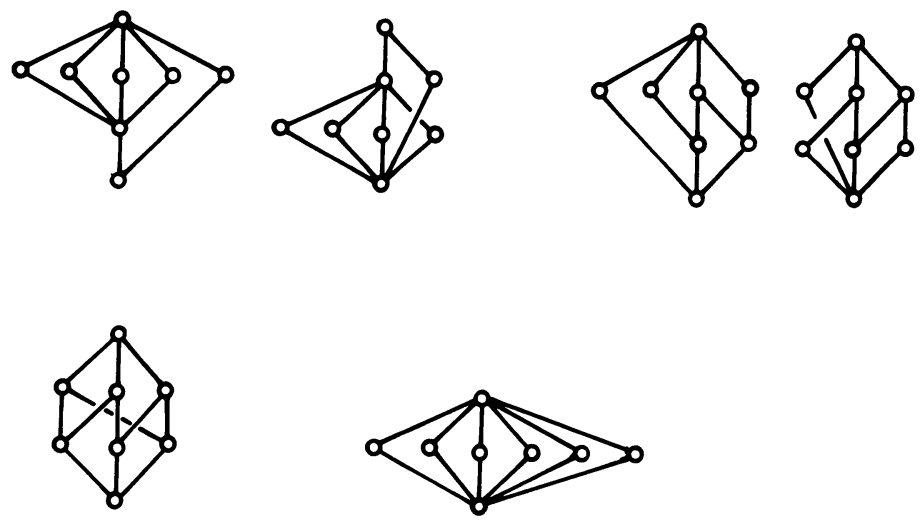

Department of Technology Tohoku Gukuin University Tagajo, Miyagi, Japan 985

1. G. BIRKHOFF, Lattice Theory, 3rd ed., Amer. Math. Soc. Colloq. Publ., vol. 25, Amer. Math. Soc., Providence, R. I., 1967. 